\title{
Assessing factors influencing smoking and smoking cessation within Chinese communities in the Greater Vancouver Area: A qualitative exploratory study
}

\author{
Noah Tregobov ${ }^{1,2,3}$, Iraj Poureslami ${ }^{1,2}$, Jessica Shum ${ }^{1,2}$, Niloufar Aran ${ }^{1,2}$, Austin McMillan', J. Mark FitzGerald ${ }^{1,2}$
}

\begin{abstract}
INTRODUCTION The prevalence of smoking is high within Chinese-Canadian communities and there is a lack of culturally and linguistically appropriate smoking cessation resources and services. We aimed to ascertain factors affecting smoking and smoking cessation from the perspectives of current smokers and key informants.

METHODS As part of a multistage mixed-methods study taking place from January 2013 to June 2014, a qualitative exploratory study design was conducted applying a community-based participatory research approach. Focus groups and interviews were conducted with adult (aged $\geq 19$ years) Chinese-Canadian current smokers ( $\geq 5$ cigarettes per day for the past 30 days) and interviews were held with key informants in Vancouver, Canada. A constant comparison method was applied to code data, and an inductive approach was used to identify emergent themes. RESULTS In all, 35 smokers from the target communities ( 11 female, 24 male) participated in 4 focus group sessions and 17 key informants ( 14 female, 3 male) were individually interviewed. Internal and external factors influencing smoking onset, continuation, and cessation from the perspectives of smokers and key informants were identified. Male smokers thought that the most influential factor influencing smoking initiation was social pressure, while female smokers thought that it was stress. Female smokers reported refraining from seeking cessation assistance due to a perceived negative image of female smokers within the community. Both key informants and participants indicated that involving friends and family in the quitting process may help to motivate smokers.

conclusions Smokers from Chinese-Canadian communities may benefit from linguistically appropriate and culturally relevant smoking cessation interventions that consider prevailing attitudes, perceptions, and beliefs. The knowledge gained may inform the development of future smoking cessation programs and resources for the target community, while our approach may be applicable to other ethnocultural or immigrant communities.
\end{abstract}

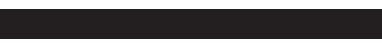 \\ AFFILIATION \\ 1 Division of Respiratory Medicine, \\ Centre for Heart and Lung Health, \\ Vancouver Coastal Health Research \\ Institute, Vancouver General \\ Hospital, University of British \\ Columbia, Vancouver, Canada \\ 2 Gordon and Leslie Diamond \\ Health Care Centre, Vancouver \\ General Hospital, University \\ of British Columbia Hospital, \\ Vancouver, Canada \\ 3 Faculty of Health Sciences, \\ Queen's University, Kingston, \\ Canada
}

\section{CORRESPONDENCE TO}

J. Mark FitzGerald. Division of Respiratory Medicine, Centre for Heart and Lung Health, Vancouver Coastal Health Research Institute, Vancouver General Hospital, 2775 Laurel Street, Vancouver, BC, V5Z 1M9, Canada. E-mail: mark. fitzgerald@vch.ca

\section{KEYWORDS}

smoking cessation, risk perception, current smokers, Chinese-Canadian communities, linguistically appropriate, culturally relevant

\section{Received: 4 May 2020}

Revised: 14 August 2020

Accepted: 21 August 2020

\section{INTRODUCTION}

Smoking and repeated exposure to secondhand smoke are two leading causes of preventable, premature morbidity and mortality worldwide ${ }^{1-3}$. China alone accounted for approximately one-third of the global smoking prevalence in $2010^{4,5}$, with $52.9 \%$ of males and $2.4 \%$ of females smoking regularly ${ }^{4}$, proportions that are reported to be increasing for men and decreasing for women ${ }^{6-8}$. Incidentally, awareness of smoking-related health risks is low in China, as three in four Chinese adults were found to have insufficient comprehension of smoking-related 
health risks ${ }^{5}$. Within China, smokers have also been found less likely to acknowledge the negative health consequences of smoking than Chinese non-smokers9. In Canada, there is a greater prevalence of smoking among Chinese immigrants compared to other Canadian immigrant communities ${ }^{10}$, and smoking is known to play an influential role within ChineseCanadian communities ${ }^{10,11}$. Differences in smoking practice and prevalence exist between males and females in Chinese-Canadian communities ${ }^{12}$, similar to China $^{4,13}$. The observed differences may be attributed to culturally rooted beliefs regarding the gender of a smoker as well as socio-environmental factors ${ }^{14}$. For instance, male smokers often obtain pro-smoking information from older male family members ${ }^{15}$, and views on self-reliance and social norms prevent full effectiveness of smoking cessation interventions ${ }^{16,17}$. In contrast to males, it is often deemed socially unacceptable for females to smoke ${ }^{18}$. These culturally influenced perceptions and gender-related differences should be considered when developing appropriate smoking cessation programs in an attempt to reduce the burden of smoking on the healthcare system, especially since Chinese immigrants currently comprise the largest ethnic minority group living in both British Columbia and Canada ${ }^{19,20}$.

In Canada, denormalization of smoking in Chinese-Canadian communities may enhance the effectiveness of cessation interventions among both adults ${ }^{17,21}$ and youth ${ }^{22}$. Enhanced consideration for socio-environmental factors and culturally influenced perceptions may enhance the effectiveness of future interventions ${ }^{23}$; however, the role that these factors and perceptions play in the promotion or hindrance of Chinese immigrants to Canada's smoking behaviours and norms is not yet fully clear. Thus, our qualitative exploratory study aimed to ascertain Chinese immigrant smokers' perceptions, attitudes, and beliefs regarding smoking as well as perceived barriers and facilitators to smoking cessation. We also collaborated with several key informants with extensive knowledge of the target communities' smoking norms. A thorough understanding of community members' and key informants' perspectives and opinions may help to inform the design and implementation of culturally appropriate smoking cessation programs for Chinese immigrant smokers and may facilitate the development of culturally relevant and linguistically accessible educational interventions and materials for the target community.

\section{METHODS}

A qualitative exploratory study design was used to ascertain factors influencing smoking practice and smoking cessation within Chinese-Canadian communities from the perspectives of adult current smokers and key informants in the Greater Vancouver Area, Canada. Ethics approval for this study was obtained from UBC Office of Behavioural Research Ethics.

\section{Participant eligibility and recruitment}

Eligible participants were adult ( $\geq 19$ years) current smokers ( $\geq 5$ cigarettes per day for the past 30 days) of Chinese descent (Mandarin- or Cantonese-speaking), who had immigrated to Canada within the past 5 years or were the children of Chinese immigrants to Canada. Key informants were healthcare professionals (e.g. clinicians, educators), immigrant and smoking cessation-related researchers, policy-/decisionmakers, or program directors of local community organizations primarily serving immigrants. Key informants had extensive knowledge of cultural norms and practices pertaining to smoking and smoking cessation, and experience working with individuals from the target communities. Recruitment efforts were greatly enhanced through professional network referrals by key informants and collaboration with community-based agencies serving immigrants (e.g. SUCCESS [United Chinese Community Enrichment Services Society] and MOSAIC), Vancouver Coastal Health community organizations, physician referrals, advertising at community events/health fairs, and the work of our bilingual research assistants and community facilitators.

\section{Study design}

A multistage mixed-methods study was conducted from January 2013 to June 2014 applying a community-based participatory research approach. This approach was applied to involve community members, key informants, and relevant community organizations throughout study design and conduct to ensure enhanced consideration for the target communities' cultural and linguistic needs. Prior to study commencement, 4 bilingual (English- and 
Mandarin- or Cantonese-speaking) community facilitators ( 2 males and 2 females) and 4 bilingual female research assistants were hired to recruit participants and to conduct interviews and focus groups; in addition, research assistants translated focus group audio-recordings from Mandarin or Cantonese to English verbatim. Both the community facilitators and research assistants were trained on approaches to participant recruitment, how to obtain informed participant consent, and how to conduct focus group sessions in a culturally relevant (with respect for cultural norms and practices) and linguistically appropriate (in a language accessible to all participants) manner to create a safe environment where participants could share their often personal thoughts and perspectives. Initially, a qualitative exploratory study was conducted with adult current smokers from the target communities and key informants. Focus groups were held with adult smokers from the target communities to ascertain perceptions, attitudes, and beliefs regarding smoking behaviours and practices, as well as to identify incentive factors or barriers to reduce or quit smoking. Key informants were interviewed or completed a survey interview on why individuals from the target communities began to smoke, the challenges facing individuals to quit, and culturally appropriate best practices to facilitate the cessation process. The perspectives and opinions of participants and key informants, and qualitative information obtained in a previous study ${ }^{23}$, served to inform the development of a study measurement tool in the form of a questionnaire that underwent validation and was applied in a cross-sectional study to assess beliefs and risk perceptions regarding smoking practices and cessation from the target communities. This paper summarizes the qualitative exploratory study's findings from the initial participant focus groups and key informant interviews. The development process of the measurement tool and the quantitative results of the cross-sectional study are reported elsewhere ${ }^{11}$.

\section{Focus group procedures and data collection}

Focus groups were held with adult current smokers from the target communities and facilitated by a team of trained bilingual research assistants and community facilitators. Prior to consenting to participate, each group was provided details regarding the study (e.g. rationale, objective, etc.), what their participation entailed, and informed that the discussion would be audio-recorded for verbatim transcription and that any responses would be anonymized. Participants were then given an opportunity to ask questions, and, if they agreed to participate, signed a written consent form in their preferred language (available in English, Chinese Simplified, and Chinese Traditional). Focus groups were held in Mandarin, or Cantonese, and followed a semi-structured format in which research staff would pose a question or prompt and allow for subsequent discussions to take place. Participants were asked to share their thoughts and views on a number of environmental, cultural, and personal factors influencing smoking onset, continuation, and cessation. For example: 'Have you tried to quit? If yes, what motivated you to quit?'. Please see Supplementary file, Document 1 , for a full list of focus group questions and prompts. Focus groups lasted from 60 to 90 minutes. In addition, research assistants completed observation notes to help contextualize responses when being reviewed during analysis. At the end of each session, modest incentives were provided to each participant for compensation of time, travel, and parking expenses.

\section{Interview procedures and data collection}

Individual interviews were conducted with key informants, who were professional individuals/ clinicians who had experience working with immigrants from the target communities, conducting research in smoking-related fields, or providing services to members of Chinese communities in the Greater Vancouver Area. Interviews were held inperson or interview questions were provided online in a typed survey format to those unavailable for an in-person interview. In-person interviews were conducted by one member of the research team who initially provided the key informant with the same study- and confidentiality-related information detailed in the 'Focus group procedures and data collection' section, allowed for any questions to be answered, and subsequently obtained written consent. For online survey interviews, key informants were provided the written consent form, provided the study team's contact information in case of any questions prior to participating, and electronically signed the consent form. In-person interviews followed a structured 
format in which a question was posed, the key informant would respond, and the interviewer would then proceed to the next question. The online survey consisted of the same open-ended questions as those posed during the in-person interviews. Key informants responded to open-ended questions regarding factors influencing smoking patterns, facilitators or barriers to quitting smoking for members of ChineseCanadian communities, and practical components of and approaches to a smoking cessation program for the target community. For example: 'Based on your experience, why do the people in your community start smoking (what causes them to pick up their first cigarette)? How do they start? - exposure, age groups, gender, etc.'. Please see Supplementary file, Document 2, for a full list of key informant interview questions and prompts. The key informant in-person interviews lasted between 30 to 45 minutes and were audio-taped for transcription, whereas online survey interviews were completed and returned to the research team. Research assistants also recorded observation notes during in-person interviews to allow for cross-referencing to contextualize quotes during data analysis. Key informants did not receive any compensation for participating.

\section{Data analysis}

Verbatim transcripts from group discussions and interviews, and typed responses to online survey interviews were systematically reviewed by two members of the research team (N.T. \& J.S.) and cross-referenced with observation notes to provide additional context/background. An inductive approach to analysis was taken that employed a constant comparison methodology to coding, an approach in which data were manually coded and assigned to a best-fit node. If coded data could not be assigned to a node, a new node was created. The coders consistently reviewed and discussed codes, nodes, and categorizations. When disagreements occurred, another member of the research team (I.P.) reviewed the coded data, provided input, and helped mediate subsequent discussions to facilitate an agreement. Following establishment of nodes, thematic analysis took place through individual categorizations by N.T., I.P., and J.S, and subsequent discussions occurred to identify emergent themes across nodes. Constant comparison of emerging themes took place until no new themes emerged. Descriptive saturation was achieved when additional nodes and themes did not emerge from further analysis.

\section{RESULTS}

Thirty-five adult Chinese-Canadian current smokers (11 female and 24 male) participated in 4 focus group sessions. In addition, seventeen key informants (14 female and 3 male; 9 in-person interview and 8 online survey interview) were interviewed. Three central themes emerged from data analysis: 1) factors influencing smoking initiation; 2) factors affecting the continuation of smoking; and 3) factors promoting/ hindering smoking cessation. The identified factors were then separated into internal (e.g. beliefs, perceptions, attitudes) and external (e.g. environment, family, work) to capture the nature of the influence on participants. The following sections provide direct quotes from participants and key informants. (IP) is used to denote data obtained from key informants through in-person interviews, while (OS) is used to denote data obtained from key informants through online survey interviews.

\section{Internal factors influencing smoking initiation,} continuation, and cessation

\section{Smoking initiation}

\section{Image}

The 'image' of a smoker was mentioned by participants as 'an adult' or more 'mature' within their communities. A Cantonese female mentioned that within her community:

'There is like a common [idea] that if that person smokes, then he or she is chill.'

One female key informant (OS) agreed and explained a common misconception of smoking within the target community:

'For women, adolescents and young adults, they pick up smoking mostly due to the misconception that smoking is a symbol of personal freedom and is associated with independence and charisma.'

However, whereas participants described the 'role model' image of smokers with reference to individuals who they knew, key informants perceived the image of smokers to be based on the media's portrayal. A female key informant (OS) detailed:

'Many [Chinese] movies and TV shows portray smoking. as the "norm", which sends the message "everyone does it".' 


\section{$\underline{\text { Stress }}$}

Stress (home/family, school, workplace, immigration, relationship) was identified by most participants and key informants as a primary factor for smoking onset and continuation. Participants and key informants both described smoking as a way to relieve stress. Immigration stress played a role in participants' smoking onset. From the experience of a male key informant (OS):

'Social changes and stresses caused by urban migration are associated with smoking initiation, especially in rural-urban migrant workers.'

\section{Smoking continuation \\ Gultural norms/practices}

Participants and key informants agreed that smoking practices differed greatly between Western culture and the Mandarin and Cantonese cultures. A Mandarin male mentioned:

'In China, people smoke everywhere, so there is little motivation to quit smoking.'

Likewise, a female key informant (OS) agreed:

'[In the] Chinese culture it's impolite to say no when others offer you a cigarette at a group gathering.'

However, whereas participants primarily described smoking as a common day-to-day practice (e.g. sharing as a gift, smoking with friends), key informants had a more holistic view and focused on the role it plays in one's perceived 'status', and the intergenerational manner in which it is passed down as a norm through 'descending belief'. A female key informant (OS) noted:

'Perhaps for the older men from mainland China, smoking means a higher social status and the financial ability to afford cigarettes.'

\section{Stress}

Stress was also mentioned as one of the main reasons for participants continuing to smoke, especially stress related to adjusting to a new culture or social environment. A Mandarin male expressed:

'Because there are language barriers...there is a lot of stress as you cannot communicate with others, which is why you smoke.'

In addition, a Mandarin female indicated:

'For me, it was a way of relaxing, and releasing. stress. I usually smoke one cigarette outside when I have a bad mood.'

\section{Smoking cessation}

Culturally-based gender differences

Participants and key informants agreed that in Chinese communities there is a cultural disapproval towards females smoking, and mentioned this stigmatization as a potential barrier to accessing cessation resources. A male key informant (IP) indicated:

'Traditionally, Chinese women did not smoke since it was socially unacceptable, especially in public.'

The participants also mentioned the influence of these perceptions to create a primarily male smoking culture. A Mandarin female added:

'Chinese people usually don't like girls to smoke... it's more acceptable for boys to smoke.'

However, whereas participants solely focused on the negative perceptions of female smokers, some key informants mentioned that smoking may be useful as a tool for women to succeed in a patriarchal business environment. A female key informant (IP) indicated:

'To triumph in a male dominant society, smoking is an effective way to network and prove their worth.'

Additional quotes regarding internal factors from participants and key informants can be found in Tables 1 and 2, respectively.

\section{External factors influencing smoking initiation, continuation, and cessation}

Smoking initiation

Family influence

Participants indicated that family members had influenced their smoking onset, and that smoking was a common activity amongst relatives. A Cantonese male stated:

'My brother-in-law taught me how to smoke. I was young, and I thought it was interesting.'

A Mandarin female added:

'Since I was born, my father was [a] heavy smoker and I am kind of growing up [sic] in the second hand [sic] smoking.'

A female key informant (OS) noted that within this community:

'Usually first-time smokers start relatively young... they get their cigarettes through friends or older siblings who smoke.'

Both participants and key informants agreed that the pressure of trying to seem 'mature' in front of older family members influenced the initiation of smoking. 
Table 1. Internal factors influencing smoking and smoking cessation, from the participants

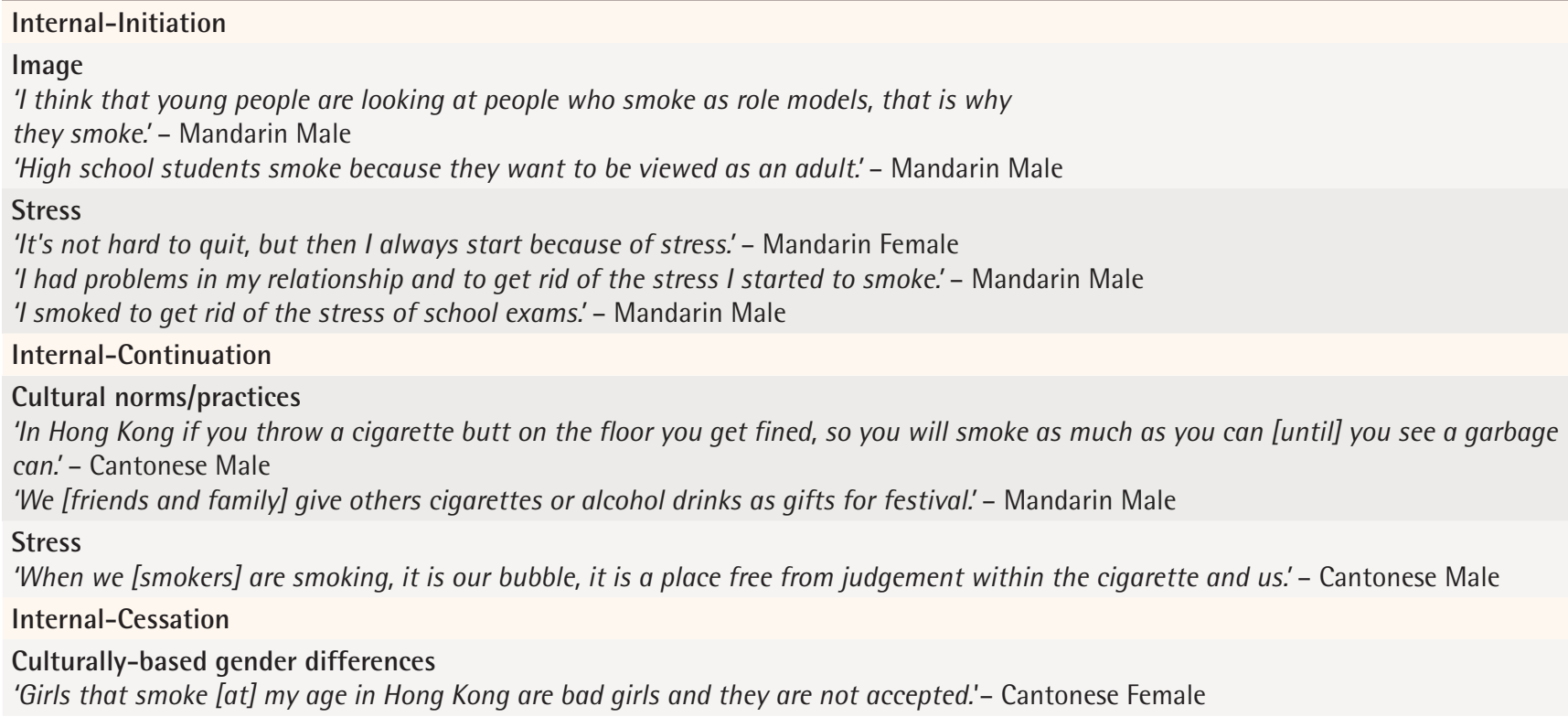

Table 2. Internal factors influencing smoking and smoking cessation, from the key informants

\section{Internal-Initiation}

\section{Stress}

'Pressure and stress from one's work and lifestyle also contribute a lot to why people would start smoking; it is seen as a stress reliever.' - Female Key Informant (OS)

Internal-Continuation

Cultural norms/practices

'Cultural acceptance and the descending beliefs of smoking appears to be the biggest reason for Chinese smokers to continue smoking even though they know smoking isn't good.' - Female Key Informant (IP)

'Chinese clients I [have] met, they started smoking when they were in their home country usually at a young age. My observation is they are mostly men.' - Female Key Informant (OS)

\section{Stress}

'I have several family friends who tried to quit but picked up the habit again for just one life-low or stressful moment. So, ongoing emotional support for this group is especially important, especially when they tend to be reluctant to share their problems.' - Female Key Informant (IP)

'When you talk with young smokers, a lot of people use the words stressed - smoke when stressed... [we must] work on teaching people ways to do deal with their stress other than light a cigarette and smoke it [sic].' - Female Key Informant (IP)

\section{Internal-Cessation}

\section{Culturally-based gender differences}

'Beauty concerns could be a good motivation for them [women] to quit smoking too. They are probably the easiest 'target group' for smoking cessation.' - Female Key Informant (IP)

'The increase of women smokers can be related to fitting in or even competing with their male peers. Due to the ethos of gender equality and the one child policy in China, women in China have become more career driven.' - Female Key Informant (IP)

\section{Social pressures}

Peer pressure and the social influence of other smokers were mentioned by both groups as reasons why participants initially gave smoking a chance, coupled with the desire to 'fit in' with a group of colleagues or friends. A Cantonese male stated:

'Most of my friends and co-workers in Hong Kong. smoked, to be a part of them, I smoked too.' 
One Cantonese female mentioned:

'A lot of my friends smoke [d] and they encouraged me to try it.'

A female key informant (OS) explained the culture's distinct pressure to appease one's elders:

'It is disrespectful to say "no" if an older generation past you a cigarette [sic].'

\section{Work environment}

Participants and key informants claimed that the work environment played a role in smoking onset. One Cantonese male stated:

'I worked in a restaurant. In order to go on break, I had to go on [smoking] breaks.'

Key informants keyed in on smoking in a business environment as a way to establish 'common ground'. A female key informant (OS) noted:

'Smoking can be used to establish relationships... [in Chinese culture] it eventually becomes a form of socializing between bosses, workers and/or clients.'

\section{Smoking continuation}

Socialization tool

Smoking played a key role in individuals' social lives, and participants mentioned that smoking served as a common ground within the culture. A Cantonese male stated:

'If you don't smoke then there is no common interest to talk about.'

A male key informant (OS) echoed this sentiment:

'Smoking has been deeply integrated with Chinese social interactions through the acceptance of cigarettes as a traditional Chinese gesture of good will.'

Participants and key informants agreed that it is a social tool, and a female key informant's (IP) perception of social smoking was concordant with many participants' views:

'Social smokers do not see a point in quitting - they feel that quitting smoking would make them lose a part of their social life.'

\section{Smoking cessation}

\section{Government and regulations}

Some participants suggested that the Canadian government should increase anti-smoking rules and regulations. One Cantonese male suggested a law in which:

\footnotetext{
'...whoever calls someone to smoke with them should
}

be fined money.'

A Mandarin male had different thoughts, stating:

'The problem is that smoking is not something. illegal.'

In addition, a participant suggested stopping the production of cigarettes. In contrast, key informants took a broader approach to enforcement. A female key informant (OS) noted:

'One of the ways to help smokers quit is establishing. various obstacles. For example, passing laws and legislations and enforcing them to maintain smoke-free environments in restaurants and other public areas.'

Key informants also focused on the importance of education as opposed to passing anti-smoking laws. For example, a male key informant (OS) noted:

'Efforts involving school-based anti-tobacco education, mass-media campaigns and community events should be made to raise public awareness of the health hazards related to tobacco smoking and SHS (secondhand smoke), and to alter misconceptions as well.'

Educational materials

Available smoking cessation resources and educational materials were deemed insufficient by some participants and key informants. Most of these individuals believed that this problem is more severe in China than in Canada. One female key informant (IP) noted:

'Not many Chinese educational materials [are] available to Chinese smokers.'

A female key informant (IP) elaborated:

'Smoking is not a disease that can be treated by takecure action. It involves a systematic action plan and continual behaviour modifications in smoking cessation.'

A few participants suggested that access to information or resources could be provided via social media platforms.

\section{Family affection}

Participants and key informants indicated that support and motivation from family members could facilitate cessation. Both groups mentioned that smokers would primarily be motivated to quit because of the harm done to loved ones (e.g. children, spouse, relatives), primarily through secondhand smoke. A Cantonese male participant stated:

'If I focus on my kids then I would think about [the] big impact smoking [has] on the health of my kids.' 
Likewise, a female key informant (IP) believed that:

'We need to involve different stakeholders of the smoker in successful cessation. Examples would be family members, close friends, mentors, physicians... covering all aspects of smoking.'

Additional quotes regarding external factors from participants and key informants can be found in Tables 3 and 4, respectively.

Table 3. External factors influencing smoking and smoking cessation, from the participants

\section{External-Initiation}

Family influence

'I started smoking because my family smoke[d].' - Mandarin Male

Social pressures

'My friends smoke[d], so I followed them. It's easier to fit in and we would have more common topics [of conversation].' - Mandarin Male

'I started to smoke socially with my friends and not really because of stress.' - Mandarin Female

Work environment

'I began to socialize at the time that I was learning how to cut hair. All hairstylists who are male like to smoke. When we were young, we wanted to fit in.' - Cantonese Male

'The US army provided each participant [with] free cigarettes.' - Mandarin Male

External-Continuation

\section{Socialization tool}

'I am not addicted to it [smoking]. It's just a social tool for me.' - Mandarin Male

'When I introduce things to [new] clients, it's like a conversation starter and takes away the barrier to meeting people.' - Mandarin Male

'Having a chat and smoking, it is always good conversation.' - Cantonese Male

\section{External-Cessation}

Government and regulations

'You cannot send all smokers to the jail, right?' - Mandarin Male

'I think the most effective way [to get smokers to quit] is to stop producing cigarettes.' - Mandarin Male

Educational materials

'I watched [a] smoking cessation video in high school and I stopped smoking after then. The video showed a picture of the lung of a smoker...it's very shocking and scaring.' - Mandarin Female

'One time I saw a video clip of black lungs and that made me want to quit at that moment, but after that I smoked again.' - Cantonese Male

Family affection

'An effective way [to quit] is to use family affection. Showing smokers the harm they bring to their families and friends, can be better than showing them the harm to themselves.' - Mandarin Male

'[Smokers] don't feel guilty for themselves, but for their families.' - Mandarin Male

\section{Table 4. External factors influencing smoking and smoking cessation, from the key informants}

\section{External-Initiation}

Family influence

'People who start smoking at younger age are usually influenced by the family members, such as trying to imitate what their parents do, or under peer pressure - just try to fit into the group and look mature.' - Male Key Informant (IP)

Social pressures

'Most participants start experiencing with their first smoke in grade 12... most start smoking more in first year university due to stress and social reasons.' - Female Key Informant (IP)

Work environment

'Smoking is habitually used to establish common grounds between co-workers.' - Female Key Informant (OS) 
Table 4. Continued

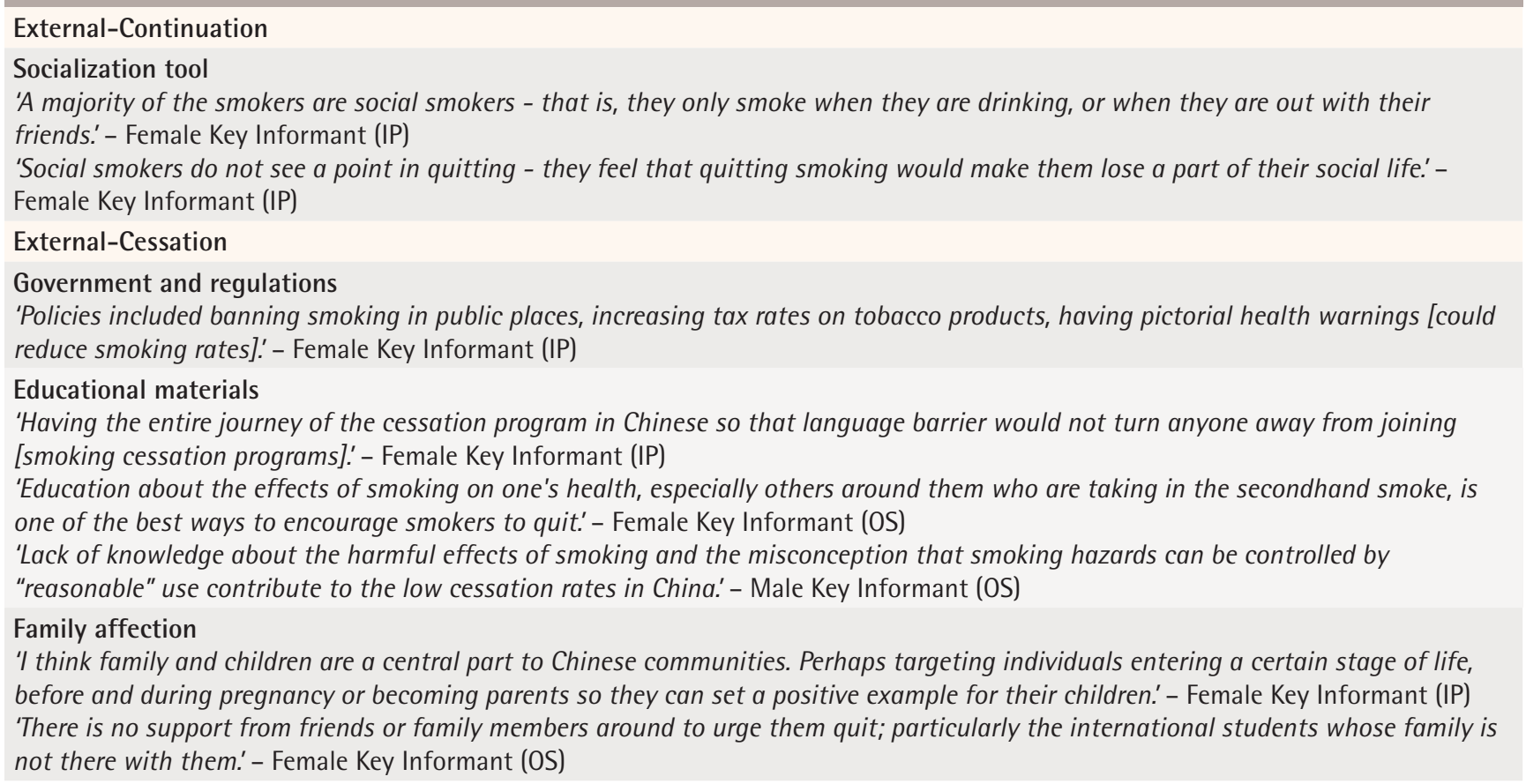

\section{DISCUSSION}

To our knowledge, this is the first qualitative exploratory study to examine both internal and external factors influencing smoking patterns and behaviours among Chinese adult smokers in Canada. We identified relationships between socio-cultural or environmental factors and smoking patterns and behaviours. The most influential internal factors identified in our study were the social pressure from peer groups and family to smoke (primarily for male smokers), followed by stress as a dominant predictor of smoking for both genders. Participants also indicated that stress was often immigration-related (e.g. language and cultural barriers). Participants and key informants agreed that culturally rooted differences in smoking practice exist between males and females. We found that many male smokers had been pressured by an older male family member to begin smoking during their teenage years; however, they continued smoking to socialize. In contrast, female smokers often acted as 'secret smokers' due to a perceived negative cultural attitude towards them, which may have acted as a barrier to accessing smoking cessation resources or programs. Overall, smoking was perceived by both males and females as a socialization tool for males, and was commonly used to find a 'common ground' with others in both business and leisure settings. There was a general sentiment amongst participants that the Canadian government takes greater action against public smoking than the Chinese government, and as a result, they were less inclined to continue smoking in Canada. Both participants and key informants indicated that there exists a lack of smoking cessation materials and resources tailored to immigrants living within Chinese-Canadian communities.

Similar studies have evaluated Chinese-Canadian smokers' perceptions and behaviours related to smoking and smoking cessation. Chinese female smokers have reported smoking in secret due to a generally negative perception in Chinese culture $^{18}$. The perception variance between the gender of a smoker was one of our critical findings. Participants and key informants indicated that within Chinese-Canadian communities there is a stigma around female smokers; however, where participants almost exclusively highlighted the perceived negative aspects of female smoking behaviours, key informants also included that 
it may serve as a socialization tool in a maledominated business setting. Thus, females may refrain from seeking cessation assistance, or feel ashamed to share their cessation struggles, even with healthcare professionals. Increased efforts from the research community to further bridge the gap in understanding of Chinese female smoking behaviours and perceptions may facilitate an increase in gender-accessible support from care providers.

A self-perpetuating cycle exists between elder and youth male Chinese smokers. Studies suggest that having parents and siblings who smoke is a predictor for an individual to smoke ${ }^{24}$. Participants and key informants indicated that cyclical learning and obeying is a trans-generational process that often promotes a general cultural acceptance within Chinese communities. Moreover, family members, especially siblings, have been reported as a direct source of cigarettes for youth and facilitators of smoking behaviour among younger individuals ${ }^{25,26}$. Participants and key informants agreed that family members were influential in smoking onset and perceived younger male family members to be under pressure to smoke in order to appear more 'mature'. They also agreed that positive influence from family members and a desire to reduce the harm of secondhand smoke on loved ones could play a preventative role in smoking onset or motivation to quit. Cessation professionals, therefore, might consider involving family members in a supportive capacity in smoking cessation programs.

Teenage or young adult members of the Chinese community have been found to be more prone to the peer pressure or social influence to smoke ${ }^{27,28}$, an external factor also identified by both participants and key informants. The perceived image of a smoker was that of being more 'adult' or 'mature'; however, participants expressed their image of a smoker with reference to individuals they knew, whereas key informants believed that smokers from the target community based their image of a smoker on the media's portrayal. Therefore, care providers and those working in smoking prevention should consider this difference in perception when incorporating social influence into cessation or prevention programs. In addition, Yang et al. ${ }^{28}$ found that around two-thirds of their study's participants smoked with others, perhaps indicating the importance of social situations and peer influence in promoting smoking behaviours within Chinese communities. This finding was corroborated by participants and key informants, who implicated a desire to 'fit in' as a reason for youth to begin smoking.

An increased emphasis on not smoking in public could promote cessation for Chinese immigrants, an important issue reported by other studies ${ }^{29-32}$. Canada's Tobacco Act prohibits the sale of tobacco products to youth (under 18 years of age) ${ }^{33}$; however, participants believed that the Canadian government should implement stricter antismoking regulations, as extreme as banning the production of cigarettes. Key informants proposed an alternative approach involving education and greater understanding of risk as opposed to increasing barriers to access. From our study and many others, we have learned that placing warning messages on smoking packages is less effective ${ }^{34,35}$, especially for younger smokers ${ }^{36}$, and direct education on the harms of smoking is not always effective ${ }^{37}$. Therefore, anti-smoking policies may consider stricter amendments and educational components. With regard to education, changing the cultural attitudes and perceptions towards smoking may require increased access to quality smoking cessation resources. Educating and empowering individuals with risk perception skills ${ }^{38,39}$, in particular youth, on the health risks associated with smoking might be a step towards decreasing smoking rates within the target communities. In addition, because smokers from the target community often start due to a perceived social/cultural norm, smoking cessation efforts may see greater effectiveness by implementing a group-based approach rather than individual quitting attempts; however, cessation professionals should continue to provide cessation support based on the individual's reasons for smoking and motivations to quit.

\section{Strengths and limitations}

The main strength of this study was the communitybased participatory research approach. The perspectives of current smokers from the target community and key informants, both of whom had an understanding of Chinese-Canadian communities' norms and practices pertaining to smoking and smoking cessation, were captured. In addition, 
the bilingualism of the community facilitators and research staff may have enhanced the communication between smokers during focus groups and created a safe environment for smokers to share their opinions. However, the generalizability of our study's findings to Chinese immigrant smokers in the Greater Vancouver Area is limited due to the use of convenience sampling. Next, as focus groups were held with multiple individuals, social desirability biases may have influenced the participants' responses. Although data were captured from 2013-2014, this study's findings are likely still applicable to the target population in 2020, as health and smoking behaviours, which are influenced by culturally rooted factors, have been found to be intergenerationally persistent among Chinese smokers ${ }^{15,40}$. Additionally, more recent studies have identified similar culturally ingrained beliefs about smoking practice and cessation among Chinese-Canadian immigrant groups ${ }^{16,21}$, as well as other Chinese immigrant groups ${ }^{17,41}$. The constant comparison methodology applied during data analysis was reliant on the study team members' subjectivity; however, triangulation between researchers and triangulation of data worked to mitigate this potential weakness. Finally, as some key informants were unavailable for in-person interviews, they responded to the interview survey. There may be inherent variability in how these individuals responded to questions compared to their response if they had completed an in-person interview.

\section{Future initiatives}

Our study's findings may serve to direct and inform smoking cessation programs for Chinese immigrant smokers to Canada. Participants and key informants communicated the existence of culturally rooted perceptions regarding smoking and smoking cessation within Chinese-Canadian communities; therefore, it is critical that future initiatives apply a culturally relevant and linguistically appropriate approach to smoking cessation. At times, participants and key informants expressed differing viewpoints on factors affecting smoking onset, continuation, and cessation; therefore, it may be beneficial to consider the viewpoints of both when developing prevention or cessation programs and resources. In addition, the identified lack of available resources for members of the Chinese immigrant community highlights the need for community-based resources that are developed with input from key stakeholders and community members. During the development of future interventions, researchers and smoking cessation professionals should consider embracing approaches that focus on attitude, perception, and belief modification. Future interventions may also provide enhanced focus on the long-term consequences of smoking and may do so through a risk perception model of smoking cessation. Table 5 contains a collection of practical recommendations for future smoking cessation programs for the target communities based on the thoughts, ideas, and values

\section{Table 5. Practical recommendations for future research programs and interventions}

\footnotetext{
We recommend the following steps be considered when helping smokers to develop and initiate a smoking cessation plan:
}

- Help smokers find a strong, motivating reason to quit smoking (e.g. quitting smoking would minimize the health consequences for them and their family members and help them to stay active, etc.).

- Promote a healthy lifestyle that is both achievable and conducive with Canada's image and norms (e.g. casual smoking may occur more frequently in China than in Canada).

- Complete simple medical tests (e.g. spirometry, carbon monoxide test) as a way to provide smokers with real evidence of the effects of smoking.

- Increase the accessibility to and quality of smoking cessation educational materials in both Chinese Traditional and Chinese Simplified. The materials should not only teach the disadvantages of smoking, but should also touch upon different aspects of life to help quit smoking as a whole (e.g. exercise, stress relief, distraction). These materials should be widely distributed, including in physicians' offices, hospitals, clinics, community agencies and community gatherings/forums. Additionally, individuals may be more receptive to educational materials if they are obtained from a trusted source, which undoubtedly varies by person (e.g. community agency, physician, etc.).

- Help smokers to develop a support system. Encourage smokers to find a non-smoking alliance who they can talk with when they are struggling or putting themselves in potentially compromising situations (e.g. having a couple drinks at a party and craving to smoke). 
Table 5. Continued

- Help to change their perceptions and misconceptions about smoking. For example, younger smokers may tend to feel invincible and fail to foresee the immediate danger while older smokers might feel it is too late to quit. Modify perception-modification approaches to best fit the needs of the individual based on age, education level, gender, language, background, etc. Essentially, there should be different curricula for each gender and age group, as different groups have different smoking habits - one size does not fit all.

- Actively consider internal and external factors that may influence a smoker's journey to quit. Guide and mentor smokers to come up with internal and external reasons to quit smoking as well as potential hinderances to acknowledge and avoid (e.g. drinking may lead to smoking).

- Quitting smoking requires a strong will/mentality. It is our experience that smokers from Chinese communities often neglect the significance of mental factors when quitting smoking. Create an environment conducive to smokers being able to share their thoughts and feelings.

- Use culturally safe/appropriate terms throughout program protocol and in educational materials (e.g. calling the smoking cessation professionals 'experts' and not 'counsellors'). From our experience, individuals of Chinese communities tend to have stigmas towards titles like 'counsellor' or other mental health vocabularies because it signifies something negative. Culture and language are very important to ensure proper communication. Smokers may feel empowered and supported throughout their journey by an 'expert' as opposed to insecure or needing help from a 'counsellor'. The importance of language and the culturall-based stigmas of certain words must not be understated.

presented by key informants during interviews and modified to fit the needs expressed by the participants during the focus groups.

\section{CONCLUSIONS}

This study ascertained internal and external factors influencing Chinese immigrant smoking and smoking cessation practices from the perspectives of current adult smokers and key informants. Smokers from Chinese-Canadian communities may benefit from linguistically appropriate and culturally relevant smoking cessation interventions. To enhance the effectiveness of smoking cessation interventions for Chinese-Canadian immigrant smokers, interventions should consider culturally-based smoking behaviours and practices that are often influenced by cultural perceptions, attitudes, and beliefs, while maintaining a patient-centered, individualized approach. Our study's findings may inform the development of future smoking cessation programs and resources for the target community and our approach may be applicable to other ethnocultural or immigrant communities.

\section{REFERENCES}

1. Centers for Disease Control and Prevention. Smoking \& Tobacco Use: Fast Facts and Fact Sheets. https://www. cdc.gov/tobacco/data_statistics/fact_sheets/fast_facts/ index.htm. Accessed August 14, 2020.

2. Jha P. Avoidable Deaths from Smoking: A Global Perspective. Public Health Rev. 2011;33(2):569-600.

\section{doi:10.1007/bf03391651}

3. World Health Organization. Tobacco. https://www.who. int/news-room/fact-sheets/detail/tobacco. Published May 27, 2020. Accessed August 14, 2020.

4. Li Q, Hsia J, Yang G. Prevalence of Smoking in China in 2010. N Engl J Med. 2011;364(25):2469-2470. doi:10.1056/nejmc1102459

5. World Health Organization. Our work in China. http:// www.wpro.who.int/china/mediacentre/factsheets/ tobacco/en/. Accessed May 24, 2019.

6. Chen Z, Peto R, Zhou M, et al. Contrasting male and female trends in tobacco-attributed mortality in China: evidence from successive nationwide prospective cohort studies. The Lancet. 2015;386(10002):1447-1456. doi:10.1016/S0140-6736(15)00340-2

7. Koplan J, Eriksen M. Smoking cessation for Chinese men and prevention for women. The Lancet. 2015;386(10002):14221423. doi:10.1016/s0140-6736(15)00416-x

8. Liu S, Zhang M, Yang L, et al. Prevalence and patterns of tobacco smoking among Chinese adult men and women: findings of the 2010 national smoking survey. J Epidemiol Community Health. 2016:71(2):154-161. doi:10.1136/jech-2016-207805

9. Yang J, Hammond D, Driezen P, Fong GT, Jiang Y. Health knowledge and perception of risks among Chinese smokers and non-smokers: findings from the Wave 1 ITC China Survey. Tob Control. 2010;19(Supplement 2):i18-i23. doi:10.1136/tc.2009.029710

10. Chen W, Bottorff JL, Johnson JL, Saewyc EM, Zumbo BD. Susceptibility to Smoking Among White and Chinese Nonsmoking Adolescents in Canada. Public Health Nurs. 2008;25(1):18-27. doi:10.1111/j.1525-1446.2008.00676.x

11. Fitzgerald JM, Poureslami I, Shum J. Assessing beliefs and risk perceptions on smoking and smoking cessation 
in immigrant Chinese adult smokers residing in Vancouver, Canada: a cross-sectional study. BMJ Open. 2015;5(2):e006435. doi:10.1136/bmjopen-2014-006435

12. Bottorff JL, Haines-Saah R, Kelly MT, et al. Gender, smoking and tobacco reduction and cessation: a scoping review. Int J Equity Health. 2014;13(1):114. doi:10.1186/s12939-014-0114-2

13. Hu TW, Tsai YW. Cigarette consumption in rural China: survey results from 3 provinces. Am J Public Health. 2000;90(11):1785-1787. doi:10.2105/ajph.90.11.1785

14. Yu ESH, Chen EH, Kim KK, Abdulrahim S. Smoking Among Chinese Americans: Behavior, Knowledge, and Beliefs. Am J Public Health. 2002;92(6):1007-1013. doi:10.2105/ajph.92.6.1007

15. Shakib S, Zheng H, Johnson C, et al. Family characteristics and smoking among urban and rural adolescents living in China. Prev Med. 2005;40(1):83-91. doi:10.1016/j.ypmed.2004.05.029

16. Mao A, Bottorff JL. A Qualitative Study on Unassisted Smoking Cessation Among Chinese Canadian Immigrants. Am J Mens Health. 2016;11(6):1703-1712. doi:10.1177/1557988315627140

17. Saw A, Paterniti D, Fung L-C, Tsoh JY, Chen MS, Tong EK. Social Environmental Influences on Smoking and Cessation: Qualitative Perspectives Among ChineseSpeaking Smokers and Non-smokers in California. J Immigr Minor Health. 2017;19(6):1404-1411. doi:10.1007/s10903-016-0358-6

18. Sansone N, Yong H-H, Li L, Jiang Y, Fong GT. Perceived acceptability of female smoking in China: findings from waves 1 to 3 of the ITC China Survey. Tob Control. 2015;24(Suppl4):iv48-iv54. doi:10.1136/tobaccocontrol-2015-052380

19. Government of Canada. Some facts about the demographic and ethnocultural composition of the population. Statistics Canada. https://www150.statcan. gc.ca/n1/pub/91-003-x/2007001/4129904-eng.htm. Updated January 25, 2008. Accessed July 25, 2019.

20. British Columbia. Statistics: Population EstimatesTotal Populations 2010. https://www2.gov.bc.ca/gov/ content/data/statistics/people-population-community/ population/population-estimates. Accessed July 25, 2019.

21. Mao A, Bottorff JL, Oliffe JL, Sarbit G, Kelly MT. A Qualitative Study on Chinese Canadian Male Immigrants' Perspectives on Stopping Smoking: Implications for Tobacco Control in China. Am J Mens Health. 2016;12(4):812-818. doi:10.1177/1557988316644050

22. Abdullah ASM, Ho WWN. What Chinese Adolescents Think About Quitting Smoking: A Qualitative Study. Subst Use Misuse. 2006;41(13):1735-1743. doi:10.1080/10826080601006433

23. Poureslami IM, Shum J, Cheng N, Fitzgerald JM. Does Culture or Illness Change a Smoker's Perspective on Cessation? Am J Health Behav. 2014;38(5):657-667. doi:10.5993/ajhb.38.5.3
24. Leonardi-Bee J, Jere ML, Britton J. Exposure to parental and sibling smoking and the risk of smoking uptake in childhood and adolescence: a systematic review and meta-analysis. Thorax. 2011;66(10):847-855. doi:10.1136/thx.2010.153379

25. Johnston V, Westphal DW, Earnshaw C, Thomas DP. Starting to smoke: a qualitative study of the experiences of Australian indigenous youth. BMC Public Health. 2012;12(1). doi:10.1186/1471-2458-12-963

26. Subramaniam M, Shahwan S, Fauziana R, et al. Perspectives on Smoking Initiation and Maintenance: A Qualitative Exploration among Singapore Youth. Int J Environ Res Public Health. 2015;12(8):8956-8970. doi:10.3390/ijerph120808956

27. Unger JB, Yan L, Shakib S, et al. Peer Influences and Access to Cigarettes as Correlates of Adolescent Smoking: A Cross-Cultural Comparison of Wuhan, China, and California. Prev Med. 2002;34(4):476-484. doi:10.1006/pmed.2001.0996

28. Myers MG, Doran NM, Trinidad DR, Klonoff EA, Wall TL. A prospective study of cigarette smoking initiation during college: Chinese and Korean American students. Health Psychol. 2009;28(4):448-456. doi:10.1037/a0014466

29. Yang T, Peng S, Yu L, et al. Chinese smokers' behavioral response toward cigarette price: individual and regional correlates. Tob Induc Dis. 2016;14(April). doi:10.1186/s12971-016-0078-7

30. Gilmore J. Report on Smoking in Canada, 1985 to 2001. Statistics Canada, 82F0077XIE No. 01. Ottawa, Canada: Minister of Industry; 2002. https://www150.statcan. gc.ca/n1/en/pub/82f0077x/82f0077x2001001-eng. pdf?st=eQX4pV6c. Accessed August 14, 2020.

31. Health Canada, Statistics Canada. Canadian Tobacco Use Monitoring Survey (CTUMS). http://ghdx.healthdata. org/series/canada-tobacco-use-monitoring-survey-ctums. Accessed May 1, 2017.

32. Béland Y, Bélanger Y, Dufour J, et al. Comparaison méthodologique entre l'Enquête sur la santé dans les collectivités canadiennes (ESCG) et l'Enquête de surveillance de l'usage du tabac au Canada (ESUTC): Rapport interne du groupe de travail. Ottawa, Canada: Statistics Canada; 2002.

33. University of Waterloo. Tobacco Use in Canada: Patterns and Trends Supplement: Tobacco Control Policies in Canada. Waterloo, ON: University of Waterloo; 2015. https://uwaterloo.ca/tobacco-use-canada/ sites/ca.tobacco-use-canada/files/uploads/files/ tobaccouseincanada_2015_accessiblepolicysuppl_final_ final-s.pdf. Accessed August 14, 2020.

34. Li L, Borland R, Yong H-H, et al. Reported Exposures to Anti-smoking Messages and Their Impact on Chinese Smokers Subsequent Quit Attempts. Int J Behav Med. 2014;21(4): 667-676. doi:10.1007/s12529-013-9349-3

35. Elton-Marshall T, Xu SS, Meng G, et al. The lower effectiveness of text-only health warnings in China 
compared to pictorial warnings in Malaysia: findings from the ITC project. Tob Control. 2015;24(Suppl 4):iv6-iv13. doi:10.1136/tobaccocontrol-2015-052616

36. Borzekowski DLG, Cohen JE. Young children's perceptions of health warning labels on cigarette packages: a study in six countries. Z Gesundh Wiss. 2014;22(2):175-185. doi:10.1007/s10389-014-0612-0

37. Yang Y, Wang JJ, Wang CX, Li Q, Yang GH. Awareness of Tobacco-Related Health Hazards among Adults in China. Biomed Environ Sci. 2010;23(6):437-444. doi:10.1016/S0895-3988(11)60004-4

38. Savoy E, Reitzel LR, Scheuermann TS, et al. Risk perception and intention to quit among a tri-ethnic sample of nondaily, light daily, and moderate/heavy daily smokers. Addict Behav. 2014;39(10):1398-1403. doi:10.1016/j.addbeh.2014.05.002

39. Krosnick JA, Malhotra N, Mo CH, et al. Perceptions of health risks of cigarette smoking: A new measure reveals widespread misunderstanding. Plos One. 2017;12(8):e0182063. doi:10.1371/journal.pone.0182063

40. Pan J, Han W. Exploring the intergenerational persistence of health behaviour: an empirical study of smoking from China. BMC Public Health. 2017;17(1):557. doi:10.1186/s12889-017-4480-8

41. Katigbak C, Maglalang DD, Chao YY, Au H, Liang W, Zuo S. Cultural Perspectives on Tobacco Use and Cessation Among Chinese American Immigrants: A Community-Engaged Qualitative Study. J Transcult Nurs. 2019;30(4):350-358. doi:10.1177/1043659618817583

\section{ACKNOWLEDGEMENTS}

We sincerely thank all participants and key informants for helping to advance our understanding, and community organizations for participant recruitment.

\section{CONFLICTS OF INTEREST}

The authors have completed and submitted the ICMJE Form for Disclosure of Potential Conflicts of Interest and none was reported.

\section{FUNDING}

The work was supported by the Vancouver Foundation, a non-government funding agency in British Columbia, Canada (Proposal number: UNR120714).

\section{AUTHORS' CONTRIBUTIONS}

Each author has made substantial contributions to acquiring the data, and helping to write, edit, and prepare the manuscript. IP and JMF worked to design the study and NT and JS conducted the majority of qualitative data analysis. All authors approve of the submitted version and agree to be accountable for their own contributions.

PROVENANCE AND PEER REVIEW

Not commissioned; externally peer reviewed. 